\title{
Optimized Link Adaptation for DVB-S2X Precoded Waveforms based on SNIR Estimation
}

\author{
Stefano Andrenacci*, Danilo Spano*, Dimitrios Christopoulos ${ }^{\dagger}$, Symeon Chatzinotas*, \\ Jens Krause ${ }^{\ddagger}$ and Björn Ottersten* \\ ${ }^{*}$ SnT - securityandtrust.lu, University of Luxembourg \\ email: \{stefano.andrenacci, danilo.spano, symeon.chatzinotas, bjorn.ottersten\}@uni.lu \\ $\dagger$ Newtec Cy, Sint-Niklaas, Belgium \\ email: $\{d c h r\} @$ newtec.eu \\ $\ddagger$ SES S.A., Chateau de Betzdorf, Luxembourg \\ email: \{jens.krause\}@ses.com
}

\begin{abstract}
The present work deals with optimized strategies to address the issue of link adaptation (Adaptive Coding and Modulation - ACM) in a multi-user multi-beam satellite system employing precoding at the gateway side. Accordingly, the focus is on the forward link of a multi-beam satellite system employing a full frequency reuse configuration. In such an interference limited setting, the Super-Framing structure of DVB-S2X air interface (format specification 2 and 3 ) enables the use of precoding techniques. The aim herein is to investigate the Signal-to-Noiseplus-Interference (SNIR) estimation error given by the User Terminals (UTs) through the so-called P2 pilots is taken into account to optimize the ACM margins.
\end{abstract}

\section{INTRODUCTION}

In recent years, the growing demand for high data rate broadband services in satellite communications through high throughput satellite (HTS) systems has driven the investigation of advanced signal processing techniques for interference mitigation which guarantee a boost of the overall spectral efficiency with a reasonable complexity. Under these assumptions, multi-beam satellite system architectures in conjunction with an aggressive frequency re-use of the total bandwidth can be an advanced solution especially when the scarcity of the spectrum is the main limiting resource. In this context, MultiUser Multiple Input Multiple Output (MU-MIMO) techniques, namely precoding, can be implemented to account for the mitigation of the co-channel interference thanks to the new Superframe (optional feature) of DVB-S2X [4], which was designed to accomodate precoding techniques.

In the literature, some precoding techniques for satellite systems [6], [8], [9], have been studied, especially focussing on the multigroup multicast nature of a satellite link which requires to group multiple users into the same transmitted codeword. It is clear that the ModCod allocation procedure in Adaptive Code and Modulation (ACM) fashion can be challenging since the reported Channel State Information (CSI) influences the calculation of the precoding matrix. Then, this matrix affects the pre-computed SNIR used to select the proper ModCod. In fact, when CSI is not perfectly estimated, as it happens when the UTs are not able to estimate all the interferer beams, the information available at the trasmitter cannot accurately evaluate the actual quality of the link and some margins need to be used to cope with possible degradation of the actual receiver SNIR.

In [7], the authors evaluate the level of the margin in a mobile satellite scenario using the Frame Error Rate (FER) of the user teminals. In this work, we derive some margins for the Unicast and Multicast precoded systems, meaning, respectively, single or multi users per codeword transmission, using the SNIR estimation at the receiver.

The paper is structured as follows. In Section II the reference system is modelled and the used air interface described, in Section III the problem of the user selection and the ModCod allocation for the relative group is explained through a characterization of the SNIR, then, in Section IV, the SNIR estimation process used by the receivers is explained and finally in Section $\mathrm{V}$ the required link margins are derived through numerical results for both Unicast and Multicast scenarios.

\section{SYSTEM MODEL}

We consider the forward link of a multi-beam satellite system when an aggressive frequency reuse is applied. Let us assume with $N_{t}$ the number of transmitting antenna elements and $N_{u}$ the total number of users served. The analytical expression of the received signal at the $\mathrm{i}$-th user is $y_{i}=\mathbf{h}_{i}^{\dagger} \mathbf{x}+n_{i}$, where $\mathbf{h}_{i}^{\dagger}$ is a $1 \times N_{t}$ vector composed of the complex channel coefficients between the $i$-th user and the $N_{t}$ antennas of the transmitter, $\mathbf{x}$ is the $N_{t} \times 1$ vector of the transmitted symbols and $n_{i}$ is the independent complex circular symmetric (c.c.s.) independent identically distributed (i.i.d) zero mean Additive White Gaussian Noise (AWGN) measured at the $i$-th user's receive antenna.

The general block scheme of a frame-based multicast multigroup precoding transmitter is shown in Figure 1. Thus, a total of $G=N_{t}$ multicast groups are assumed, with $\mathcal{I}=\left\{\mathcal{G}_{1}, \mathcal{G}_{2}, \ldots \mathcal{G}_{G}\right\}$ the collection of index sets and $\mathcal{G}_{k}$ the set of users that belong to the $k$-th multicast group, $k \in\{1 \ldots G\}$. Each user belongs to only one group, thus $\mathcal{G}_{i} \cap \mathcal{G}_{j}=\varnothing, \forall i, j \in\{1 \cdots G\}$. With $\mathbf{w}_{k} \in \mathbb{C}^{N_{t} \times 1}$ we denote the precoding vector applied to the transmit antennas for the 


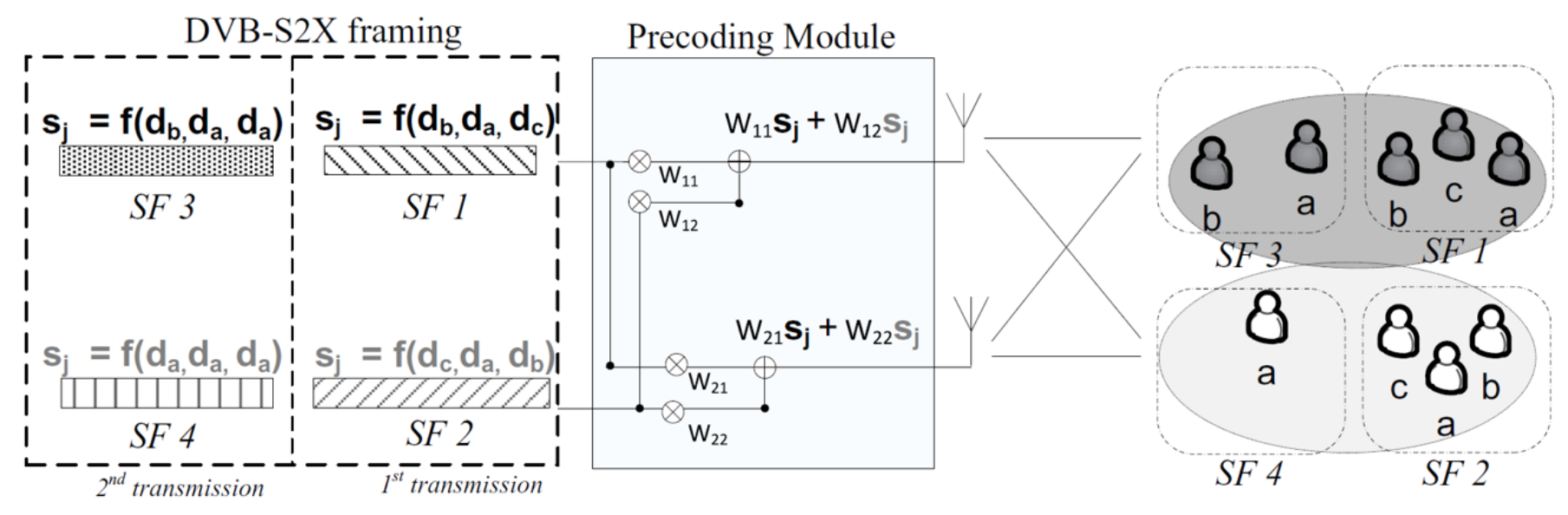

Figure 1: Frame based precoding transmitter. A symbol denoted as $s_{[i]}$ is addressed to the $i$-th user of the corresponding frame. In frames 3 and 4, different amount of data is transmitted to each user. Symbols denoted as $s_{a}$ are not carrying identical data.

$k$-th group. Let us also denote the number of users per group as $\rho=N_{u} / G$. By collecting all user channels in one channel matrix, the general linear signal model in vector form reads as

$$
\mathbf{y}=\mathbf{H x}+\mathbf{n}=\mathbf{H W} \mathbf{s}+\mathbf{n}
$$

where $\mathbf{y}$ and $\mathbf{n} \in \mathbb{C}^{N_{u}}, \mathbf{x} \in \mathbb{C}^{N_{t}}$ and $\mathbf{H} \in \mathbb{C}^{N_{u} \times N_{t}}$. The multigroup multicast scenario imposes a precoding matrix $\mathbf{W} \in \mathbb{C}^{N_{t} \times N_{t}}$ that includes as many precoding vectors (i.e columns) as the number of groups $\left(G=N_{t}\right)$.

A simplified channel model in the fashion of [8] can also be derived with the aim of providing a more tractable representation. Let us define multiple square channel matrices $\mathbf{H}_{[i]}, i=1 \ldots \rho$. Each matrix corresponds to a "a single user per beam" instance. To model the frame based precoding constraint, the general input-output signal model can be defined as [8]: $\mathbf{y}_{[i]}=\mathbf{H}_{[i]} \mathbf{x}_{[i]}+\mathbf{n}_{[i]}=\mathbf{H}_{[i]} \mathbf{W} \mathbf{s}_{[i]}+\mathbf{n}_{[i]}$ where $\mathbf{y}, \mathbf{x}, \mathbf{n}, \mathbf{s} \in \mathcal{C}^{N_{t}}$, with $\mathcal{E}\|\mathbf{n}\|^{2}=\sigma^{2}$ and $\mathcal{E}\|\mathbf{s}\|^{2}=1$, while $\mathbf{H}_{[i]} \in \mathcal{C}^{N_{t} \times N_{t}}$ is a one-user-per-beam instance of the total non-square channel matrix. The index $[i]$ corresponds to the different UTs per beam that need to be served by the same frame, i.e. $i=1, \ldots \rho$, where $\rho=N_{u} / G$ is number of users per group. Also, due to the one group per antenna assumption, $N_{u}=\rho \cdot N_{t}$.

\section{A. DVB-S2X Superframe Option}

The Annex E of the DVB-S2X standard [4] specifies the optional framing structure which enables the use of precoding techniques for the forward link of a satellite system. Basically, the superframe structure fixes the use of:

1) A constant superframe length of 612540 symbols

2) A preamble of 720 symbols which includes the Start of Super-Frame (SOSF) and the Super Format Frame Indicator (SFFI)

3) 2 different scrambling sequence definitions (Gold codes) for the so-called reference and payload data scramblers
The super-framing structure is introduced for multiple purposes, in particular for MU-MIMO techniques, namely precoding, to mitigate the interference caused by an aggressive frequency re-use.

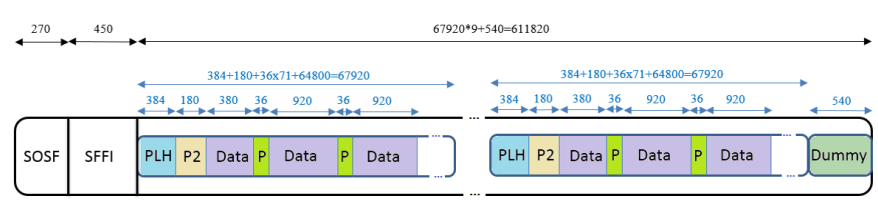

Figure 2: Framing structure of the DVB-S2X Superframe option, format specification 2

In Figure 2, the framing structure definition for the format specification 2 of the superframe is shown. In this document we focus on the precoded pilots only since the aim is the estimation of the SNIR in DVB-S2X:

- the superframe is scrambled using two different scrambling sequences: the first sequence, so called reference data scrambler, is the same for all beams and it overlays SoSF and Pilot fields. The second one, the so called payload data scrambler, is beam dependent and provides resilience to co-channel interference.

- two types of pilot fields are present: the super frame modulated and, possibly, precoded pilot symbols, called $\mathrm{P} 2$, that can be used for SNIR estimation, and the not precoded pilots, $\mathrm{P}$ for channel estimation.

As a consequence of the two previous points, for every bundle PL-frame [4] a beam-specific signature for SNIR estimation is defined, given by the combination of the fixed P2 sequence and the beam-specific payload data scrambler.

\section{Multicast Aware Scheduler}

In [2], the authors developed a multicast aware user scheduling and ModCod allocation scheme for precoded systems. The block diagram of the scheme is shown in Figure 3. 


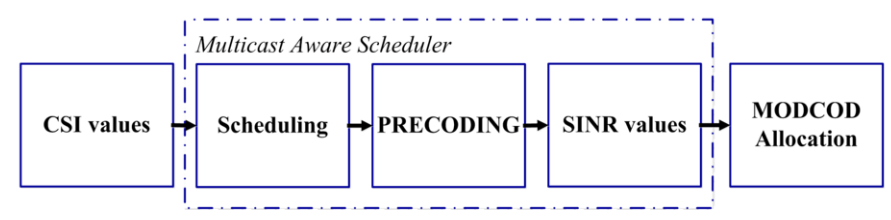

Figure 3: Multicast Aware Scheduling and Modcod allocation block scheme

Following the principles of MU-MIMO communication link, which foresee the use of an $N_{t}$ dimensional vector rather than a single SINR value, the scheduler tries to maximize the similarity of two channel vectors in the same frame through the maximization of their projections. As easily understandable, precoding is affected by scheduling and vice versa and they both contribuite to the allocation of the ModCod to be used to close the link. In [2], the authors derived a low complexity method which assumes the perfect knowledge of the CSI. In the present work we tackle the problem of having non-perfect CSI at the gateway. When this assumption occurs, the effective SNIR at the receiver can be even lower than the one used for ModCod allocation with unwanted consequences such as a link outage. To avoid this issue, an estimated SNIR based link margin has to be applied before the ModCod allocation is proposed.

The following subsection has the aim of characterize the different SNIR values which can be faced in different parts of the forward link chain.

\section{A. SNIR characterization}

The general formula for calculating the Precoded SNIR over a linear channel is:

$$
\operatorname{SNIR}_{P C k}=\frac{p_{k}\left|\mathbf{h}_{k}^{\dagger} \mathbf{w}_{k}\right|^{2}}{\sigma_{k}^{2}+\sum_{\substack{n=1 \\ n \neq k}}^{N t} p_{n}\left|\mathbf{h}_{k}^{\dagger} \mathbf{w}_{n}\right|^{2}}
$$

According to the guidelines for precoding implementation [5], this formula is applicable for either data symbols and P2 pilot symbols. We define:

- H : the effective channel between transmitters and receivers. It is the actual channel w.r.t the scheduled users, hence it is a 2 dimensional matrix amongst the transmitting antennas and the scheduled users.

- $\hat{\mathbf{H}}$ : the estimated channel through CSI estimation. As before, it is a 2 dimensional matrix.

- $\widehat{\mathbf{W}}$ : the precoding matrix calculated using CSI estimation

Based on the previous definitions we call (the index $i$ stands for the i-th user but the formulation can be applied in a vector form):

- $\operatorname{SNIR}_{P C i}(\widehat{\mathbf{H}}, \widehat{\mathbf{W}})$ : Precoded SNIR at the Gateway $(\mathrm{GW})$. This quantity is the one used for modcod allocation in precoded systems and it is based on estimated CSIT (CSI at the Transmitter).

- $\operatorname{SNIR}_{P C i}(\mathbf{H}, \widehat{\mathbf{W}})$ : Actual Precoded SNIR at the receiver. This quantity is the effective precoded SNIR at the Rx antenna, which depends on the precoding matrix $\hat{\mathbf{W}}$ and on the effective channel $\mathbf{H}$.

- $S \widehat{N I R_{P C i}}\left(f_{\text {estimAlgo }}(\mathbf{H}, \widehat{\mathbf{W}})\right)$ : Estimated precoded SNIR by the Rx. This quantity is the precoded SNIR, estimated by the Rx and it is the SNIR which drives the demapper. It depends on the estimation algorithm and it is a function of $\operatorname{SNIR}_{P C i}(\mathbf{H}, \widehat{\mathbf{W}})$.

In addition to the previous definition we introduce the SNIR related to the non-precoded fields of the super-frame structure which is:

- $S N I R_{N P C i}(\mathbf{H})$ : Non-precoded SNIR at the Rx. This quantity is the effective non-precoded SNIR at the Rx antenna. CSIT are base on this quantity.

To better clarify the role of these quantities, a general block scheme of the overall forward link structure can be found in the following figure:

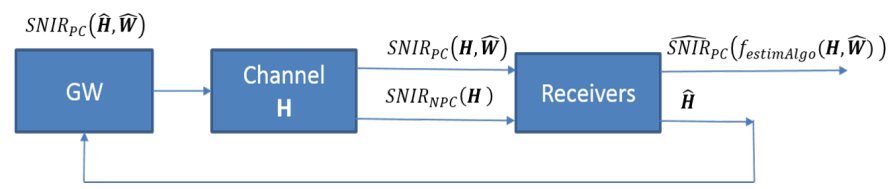

Figure 4: General block scheme on the SNIR characterization for the forward link

The Precoded SNIR is computed at the GW based on the CSI statistics estimated by the User Terminals (UTs). According to the Multicast aware scheduling scheme, CSIT are used to allocate the proper ModCod to the specific group of users. The ModCod allocation has influence on the XFECFRAME and P2 fields only while the Non-precoded fields (i.e. the SOSF and the SF-Pilot fields) remain unchanged. At the input of each receiver, the received superimposed waveform has two different SNIR depending on the specific field of the SF: the Precoded SNIR $S N I R_{P C i}(\mathbf{H}, \widehat{\mathbf{W}})$ for P2 and XFECFRAME fields and the Non-precoded SNIR $S N I R_{N P C i}(\mathbf{H})$ for SOSF and SF-Pilot fields. Due to receiver impairments, the estimated $S \widehat{N I R_{P C i}}\left(f_{\text {estimAlgo }}(\mathbf{H}, \widehat{\mathbf{W}})\right)$ can also have some mismatch w.r.t. the $\operatorname{SNIR}_{P C i}(\mathbf{H}, \widehat{\mathbf{W}})$.

\section{SNIR ESTIMATION AT THE TERMINAL}

In order to estimate the SNIR at the receiver using the P2 definition of the Superframe structure, the well-known data aided version of the SNORE algorithm [3] is here used:

$$
\widehat{S N I R}{ }_{i}=\frac{\mathbf{y}_{i}^{\dagger} \mathbf{P} \mathbf{y}_{i}}{\mathbf{y}_{i}^{\dagger} \mathbf{P}^{\prime} \mathbf{y}_{i}}, P=\frac{\mathbf{c}_{i} \mathbf{c}_{i}^{\dagger}}{N}, \mathbf{P}^{\prime}=\mathbf{I}-\mathbf{P}
$$

where $\mathbf{c}_{i}$ is the $\mathrm{i}$-th known sequence, $N$ is the length of the sequence, $\mathbf{y}_{i}$ is the $\mathrm{i}$-th received signal over the $N_{t}$ possibilities (in a single frame), $\mathbf{I}$ is the identity matrix and $\mathbf{P}, \mathbf{P}^{\prime}$ are the projection matrices onto, respectively, the useful signal and noise (plus interferer) signal.

While in previous works the algorithm has been used in noise limited channel conditions, in the present work the focus is on co-channel interference scenarios. By relying on the 
gaussian approximation for noise plus the residual interference waveforms after precoding, the DA-SNORE algorithm has shown to properly work also in the current scenario, even when multiple users per codeword are scheduled in TDM fashion. Due to the considerable length of the P2 pilot field in DVBS2X Superframe option, the reduced complexity version of the SNORE for practical implementation is used:

$$
\begin{gathered}
\widehat{S N I R}_{i}=P_{S} /\left(P_{R}-P_{S}\right) \\
P_{S}=\left\{\frac{1}{N} \sum_{m=0}^{N-1} \operatorname{Re}\left\{y_{i}[m] c_{i}^{*}[m]\right\}\right\}^{2} \\
P_{R}=\frac{1}{N} \sum_{m=0}^{N-1}\left\{y_{i}[m] y_{i}^{*}[m]\right\}^{2}
\end{gathered}
$$

where $P_{S}$ is the useful signal power and $P_{R}$ is the received signal power given by the summation of the useful signal, the noise and the residual interference.

\section{NUMERICAL RESUlts}

In the present section, we evaluate the level of margins to be used for the ModCod allocation through numerical simulations for both Unicast and Multicast scenarios. The simulation parameters used are the one listed in the following:

\section{Unicast Parameters}

- Multi-beam MMSE Precoding

- Satellite Orbit: GEO

- Full frequency Reuse

- Frequency Resource: Ka Band for Downlink

- Single Gateway

- Psat $=100 \mathrm{~W}$

- Number of beams: 71

\section{Multicast Parameters}

- Multi-beam Average Precoding

- Satellite Orbit: GEO

- Full frequency Reuse

- Frequency Resource: Ka Band for Downlink

- Single Gateway

- Psat $=100 \mathrm{~W}$

- Number of beams considered: 9 over 245 for the whole coverage

- 5 users per codeword (Frame)

In both cases, we are going to evaluate the effect on the SNIR of the non-perfect CSI at the Gateway due to nullification effects. The nullification effect is a source of degradation for precoding and it is given by the inability for practical receivers to estimate all the coefficients of the CSI vector. It happens, for example, when the pilots from a certain beam are received by the UT at very low SNR and this is usually the case for beams which are not far from the beam of interest. When the interferer waveform cannot be detected by the User Terminal, a null value is normally used to replace the correspondent coefficient of the vector. This rule applies for all the estimated vectors by the UTs and, depending on the sensitivity of the detection, the CSI matrix can be relatively different from the actual channel matrix.

For the numerical results, we considered a nullification threshold based on the $\mathrm{C} / \mathrm{I}$ value of $15 \mathrm{~dB}$, where $\mathrm{C}$ represents the power of the useful signal and $\mathrm{I}$ is the whole interferer power without the use of any interference mitigation technique. The reason why we choose a $\mathrm{C} / \mathrm{I}$ based threshold with the specified value can be found in [1], [5]. Basically, when pilots orthogonality lacks, the detectability of the CSI is mostly related to the interference level than the noise one (while the noise is predominant the other way round).

In Figure 5 we report a comparison in terms of SNIR between the calculated SNIR at the GW, the actual SNIT at the terminal and the estimated SNIR through P2 pilots for a unicast case ( 1 user per codeword). More specifically, we can focus, at first, on the dashed red curve which represents the calculated SNIR for an AWGN channel. Since we assume to have perfect channel state information at the GW (ideal CSI recovery), there is no mismatch between the CSI matrix and the actual channel matrix, therefore, the calculated SNIR at the GW and the actual SNIR at the Rx have the same values. The green curve is the estimated SNIR through P2 pilots and it basically converge ti the actual SNIR. This happens because the reported estimation is averaged over different realizations. The purple curve is the actual SNIR at the receiver when nullification impairments are affecting the CSI matrix. By comparing this curve with the black one, which is the calculated precoded SNIR at the GW in case of nullification, we can see a difference of about $0.5 \mathrm{~dB}$. There is also an additional margin of $0.2 \mathrm{~dB}$ due to the estimated SNIR as we can see from the light and dark blue curve.

In total we can assume a margin of $0.7 \mathrm{~dB}$ in this scenario to be applied at the GW before the ModCod allocation, which is required to close the link without outages. For the chosen SNIR range, this $0.7 \mathrm{~dB}$ corresponds to a margin in terms of spectral efficiency of $0.25 \mathrm{bit} / \mathrm{symbols}$, hence, about $10 \%$ of the obtained spectral efficiency.

Regarding the multicast scenario, numerical results are shown in Figure 6. The dashed red curve is obtained without nullification of CSI matrix and it is the calculated precoded SNIR at the GW for the multicast scenario. The black curve is the calculated precoded SNIR at the GW as before but when nullification effects are introduced. As we experienced in the previous figure, the calculated SNIR at the GW are very similar in the two cases and this is pretty understandable since the precoder is calculated based on the specific CSI matrix. By comparing the red dashed curve with the blue one, which is provided by the estimated SNIR at the terminal, a mismatch of about $1 \mathrm{~dB}$ can be experienced, therefore, a margin of $1 \mathrm{~dB}$ is required to avoid outages, which corresponds to 0.27 bit per symbols in terms of spectral efficiency, $15 \%$ of the obtained spectral efficiency.

\section{CONCLUSION}

In this work, the problem of the ModCod allocation for precoded systems affected by non-perfect CSI at the GW has 


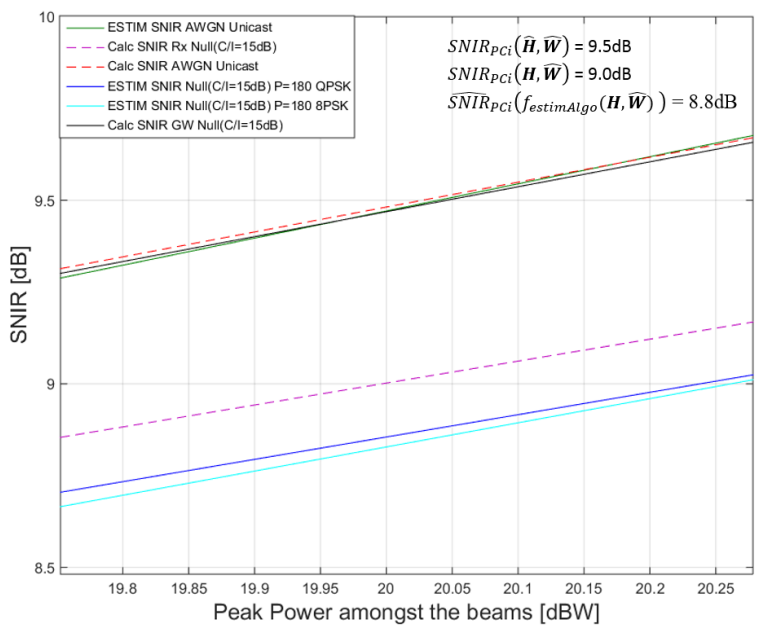

Figure 5: Numerical results of the obtained SNIR in a unicast scenario

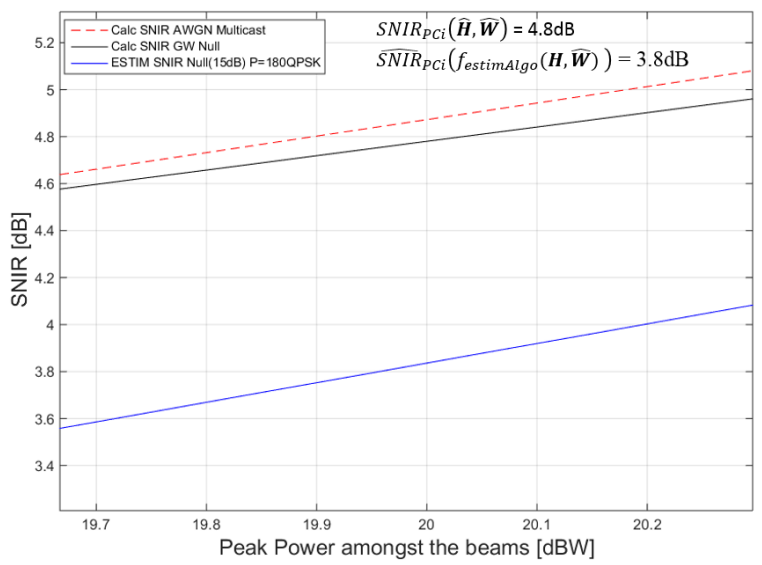

Figure 6: Numerical results of the obtained SNIR in a multicast scenario

been studied. At first, we verified that the actual precoded SNIR at the receivers can be lower then the one computed at the GW and used for ModCod allocation when nullification impairments occur. We also showed that a SNIR estimation procedure on the so-called P2 pilots contained in the Superframe structure (Format 2,3) of DVB-S2X standard, can be used to calculate margins for the ModCod allocation. For the considered Unicast scenario, the margin has been estimated to be $0.7 \mathrm{~dB}$ while for the Multicast case, the level of margin is around $1 \mathrm{~dB}$.

\section{REFERENCES}

[1] P. D. Arapoglou, A. Ginesi, S. Cioni, S. Erl, F. Clazzer, S. Andrenacci and A. Vanelli-Coralli, "DVB-S2X-enabled precoding for high throughput satellite systems," Int. J. Satell. Commun. Network., 2015.

[2] D. Christopoulos, S. Chatzinotas and B. Ottersten, "Multicast Multigroup Precoding and User Scheduling for Frame-Based Satellite Communica- tions", Wireless Communications, IEEE Transactions on 14 (9), 46954707

[3] C. E. Gilchriest 'Signal to Noise Monitoring", JPL Space Programs Summary, No 37-27, Vol IV, pp 169-176

[4] ETSI EN 302 307-2 "Digital Video Broadcasting (DVB), Second generation framing structure, channel coding and modulation systems for Broadcasting, Interactive Services, News Gathering and other broadband satellite applications, Part II: S2-Extensions (DVB-S2X).” Available on ETSI web site (http://www.etsi.org)

[5] ETSI TR 102376 V1.1.1 "Digital Video Broadcasting (DVB), Implementation guidelines for the second generation system for Broadcasting, Interactive Services, News Gathering and other broadband satellite applications; Part 2 - S2 Extensions (DVB-S2X)", March 2015, Available on ETSI web site (http://www.etsi.org)

[6] D. Christopoulos, S. Chatzinotas, and B. Ottersten, "Weighted fair multicast multigroup beamforming under per-antenna power constraints," IEEE Trans. Signal Process., vol. 62, no. 19, pp. 5132-5142, Oct. 2014.

[7] A. Rico-Alvario, A. Tato and C. Mosquera, "Robust adaptive coding and modulation scheme for the mobile satellite forward link," 2015 IEEE 16th International Workshop on Signal Processing Advances in Wireless Communications (SPAWC), Stockholm, 2015, pp. 530-534.

[8] D. Christopoulos, P.-D. Arapoglou, S. Chatzinotas, and B. Ottersten, "Linear precoding in multibeam satcoms: Practical constraints," in 31st AIAA International Communications Satellite Systems Conference (ICSSC), Florence, IT, Oct. 2013.

[9] M. Á. Vázquez and A. I. Pérez-Neira and D. Christopoulos and S Chatzinotas and B. Ottersten and A. Ginesi and D. Arapoglou and G. Taricco, "Precoding in Multibeam Satellite Communications: Present and Future Challenges", IEEE Wireless Communications Magazine,2016. 